\title{
Micropatterning Performance and Physical Characteristics of Water-soluble High Molecular Weight Polysaccharide Photoresist Materials
}

\author{
Toru Amano ${ }^{1,2^{*}}$, Makoto Kobayasi ${ }^{2}$, Satoshi Takei ${ }^{1}$ \\ ${ }^{I}$ Department of Mechanical Systems Engineering, Toyama Prefectural University, \\ Imizu, Toyama 939-0398, Japan \\ ${ }^{2}$ Gunei Chemical Industry, Takasaki, Gunma 370-0032, Japan \\ *t-amano@gunei-chemical.co.jp
}

\begin{abstract}
Pullulan, a base polymer, is a water-soluble high-molecular-weight polysaccharide that easily forms films and is used for edible films and the like. We examined whether a photoresist material that makes the best use of its film-forming property and water solubility could be realized. The hydroxyl group of pullulan was modified with a photosensitive group to the extent that water solubility could be maintained, and a photosensitive material was created. This material is applied on a silicon wafer using spin coating, exposed with a mask contact exposure device, and then developed with water to evaluate sensitivity, etching resistance, and coating film strength. Microfabrication evaluation was performed. The result may be applicable as a water-soluble micropatterning material. Because this material does not use an organic solvent or a highly toxic strong alkaline developer, it is useful as a low environmental load-patterning material.
\end{abstract}

Keywords: Micro-patterning, Photoresist, Polysaccharide chain, Water-soluble material

\section{Introduction}

Photoresist materials are indispensable in semiconductor manufacturing, as electronic devices become more integrated, smaller more reliable, and faster in signal speed. Conventional photoresist materials used in semiconductor manufacturing are synthesized from fossil raw materials such as acrylic resin and phenolic resin, coated with an organic solvent, and developed with a strong alkaline developer or organic solvent. The many resins, solvents, and alkaline developers currently create significant waste, which is a problem.

In recent years, much attention has been paid to water-soluble photoresist materials that can be applied and developed with water. The main materials are water-soluble resin and water, and these are low environmental load-patterning materials that do not use an organic solvent or a highly toxic strong alkaline developer.

Although polyvinyl alcohol, polyacrylamide, and the like are often studied as water-soluble resins, they cannot be said to have a small environmental load because they are produced from fossil raw materials. [1-8]

Saccharides have been proposed as a water-soluble resist material with low environmental load, but it cannot be said that the low environmental load is sufficient because the coating is performed using an organic solvent. [9]

In addition, a plant-derived and water-soluble sugar (dextrin) photoresist material that can be coated and developed with water has been studied, but the coating strength is low due to the low molecular weight. As a result, the film strength after UV curing is low. [10]

Therefore, a photosensitive material using a high molecular weight polysaccharide (pullulan) with film-forming properties as the base polymer leads to a new micro-patterning material with improved coating strength compared with conventional watersoluble nanopatterning material.

In this paper, to confirm effectiveness of the watersoluble micropatterning material, the hydroxyl group of pullulan is modified with a photosensitive group to the extent that water solubility can be maintained, and exposure sensitivity and etching are 
tested. We report that resistance, coating strength, and patterning properties.

\section{Experimental}

\subsection{Materials}

The high molecular weight polysaccharide used in this study was made from pullulan manufactured by HAYASHIBARA Co., Ltd.

For comparison, dextrin manufactured by San-ei Sucrochemical (NSD500) and NSD500 with the low molecular weight fraction removed (NSD500Hi, hereinafter) were used. To remove the low molecular weight fraction, dextrin was dissolved in water, and then methanol and ethanol were added to precipitate only the polymer portion in dextrin. The low molecular weight fraction removed can be arbitrarily controlled by the mixing ratio of water and solvent. The more water is, the higher the amount of low molecular weight fraction removed.

The results of gel permeation chromatography (HLC-8320GPC: Tosho) measurements to confirm the molecular weight distribution of each raw material are shown in Fig. 1 and Table 1.

The number average molecular weight $(\mathrm{Mn})$ of pullulan used as a raw material was 73292 , and the weight average molecular weight $(M \mathrm{w})$ was 161980 . For NSD500, Mn was 2454 and $M \mathrm{w}$ was 11383 , and for NSD500Hi, Mn was 7164 and $M \mathrm{w}$ was 32637 .

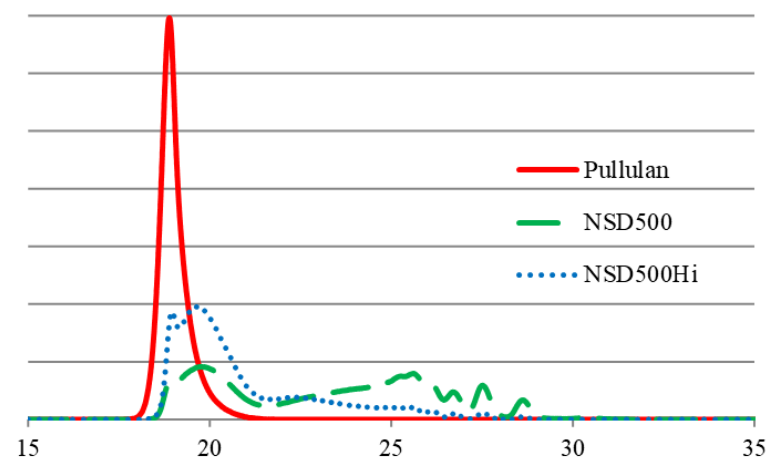

Fig. 1. Molecular weight distributions of base polymer.

Table.1. GPC measurement results of base polymer.

\begin{tabular}{|l|c|c|}
\hline & $M \mathrm{n}$ & $M \mathrm{w}$ \\
\hline Pullulan & 73292 & 161980 \\
\hline NSD500 & 2454 & 11383 \\
\hline NSD500Hi & 7164 & 32637 \\
\hline
\end{tabular}

\subsection{Synthesis of photosensitive polymer}

The base polymers (Pullulan, NSD500, NSD500Hi) are dissolved in $\mathrm{N}$-methylpyrrolidone,
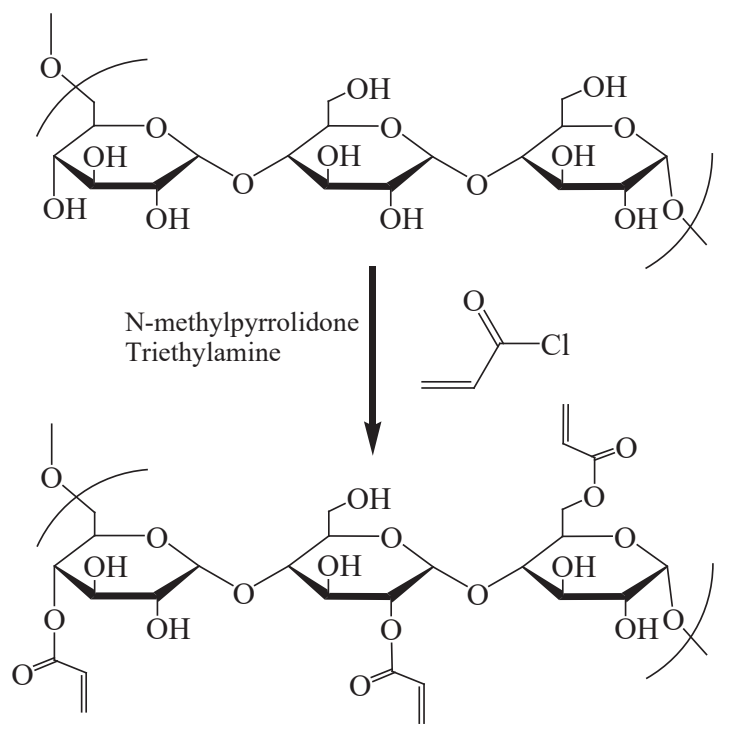

Fig. 2. Modification of pullulan hydroxyl groups to photosensitive groups.

and acrylic acid is used to denature photosensitive groups by $30 \%$ with respect to all hydroxyl groups while maintaining the system temperature at $15^{\circ} \mathrm{C}$ or lower. Chloride was weighed and dropped over 2 h.

The solutions were then aged for $2 \mathrm{~h}$ at $15^{\circ} \mathrm{C}$ or below and neutralized with triethylamine. Next, $\mathrm{N}$ methylpyrrolidone and a neutralizing salt were purified and removed, and the inside of the system was replaced with water to obtain a polysaccharidebased photosensitive material.

The modification to the photosensitive group was confirmed by ${ }^{13} \mathrm{C}$-NMR and FT-IR. As an example, the synthesized structure of pullulan is shown in Fig. 2.

Hereafter, the acrylic acid-denatured polysaccharide chains are referred to as Pullulan-A, NSD500-A, and NSD500Hi-A.

\subsection{Measurement of exposure sensitivity}

Omnirad 2959 (manufactured by IGM Resins B.V.) was used as the photosensitizer, and 1, 2, or 3 $\mathrm{wt} \%$ was added to each aqueous solution of the acrylic acid-modified base polymer.

The obtained aqueous solutions were applied to a silicon wafer, spin-coated at $3000 \mathrm{rpm}$ for $30 \mathrm{~s}$ using a spin coater (MS-B100: MIKASA) and baked at $80^{\circ} \mathrm{C}$ for $60 \mathrm{~s}$ to volatilize and remove water. Next, exposure is performed using a mask contact exposure device (LTCET-500: Lithotec Japan) and a sensitivity confirmation mask (Taiyo-ink Corporation: 5-inch Multi-Transmission Mask), the film thickness is measured, and then the film is 




Fig. 3. Sensitivity measurement process for water-soluble micropatterning materials.

developed to determine the residual film ratio. This process is diagrammed in Fig. 3. Using the above mask, it is possible to easily create a sensitivity curve.

\subsection{Measurement of etching resistance}

FA-1-TK manufactured by Samco Corporation was used to measure the etching resistance. For the measurement samples, $3 \mathrm{wt} \%$ of photosensitizer was added to each polymer. Pullulan-A was exposed to $100 \mathrm{~mJ} / \mathrm{cm}^{2}$, NSD500-A and NSD500Hi-A were exposed to $600 \mathrm{~mJ} / \mathrm{cm}^{2}$, and Cresol Novolac was used as a reference. $\mathrm{CF}_{4}$ and $\mathrm{O}_{2}$ were used as the etching gases.

\subsection{Measurement of coating film strength}

The measuring device used for coated film strength was a Dynamic Ultra Micro Hardness Tester: DUH-211S manufactured by Shimadzu Corporation.

\subsection{Confirm of microfabrication}

The evaluation method is as shown in Fig 4. A mask close contact exposure device (LTCET-500: Lithotec Japan) and a mask for microfabrication

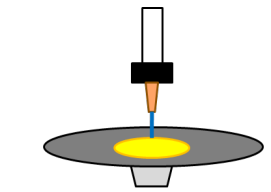

(1) Photoresist solution Drop

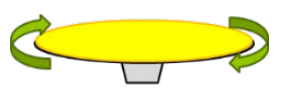

(2) Spin coat
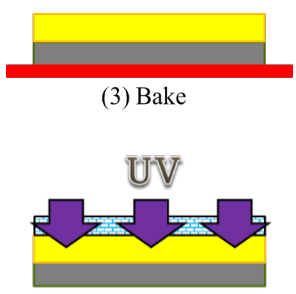

(4) Exposure $600 \mathrm{~mJ} / \mathrm{cm}^{2}$



(5) Water development

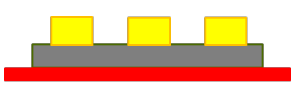

(6) Dry

Fig 4. Microfabrication process of water-soluble patterning material.

confirmation (TOPPAN PRINTING CO., LTD: TOPPAN-TEST-CHART-NO1-PN) were used to carry out the evaluations.

\section{Research and Discussion}

3.1 Exposure sensitivity evaluation

The exposure sensitivity of the water-soluble micropatterning material using high-molecularweight polysaccharides was confirmed.

Pullulan-A was exposed at $150 \mathrm{~mJ} / \mathrm{cm}^{2}$, and NSD500Hi-A and NSD500-A were exposed at $1200 \mathrm{~mJ} / \mathrm{cm}^{2}$.

The sensitivity data indicate that to obtain a residual film ratio of $80 \%$ or more, NSD500-A requires the addition of $2 \mathrm{wt} \%$ or more of photosensitizer and exposure of $900 \mathrm{~mJ} / \mathrm{cm}^{2}$ or more as shown in Fig. 5, and NSD500Hi-A requires the addition of $2 \mathrm{wt} \%$ or more of the photosensitizer and exposure of $660 \mathrm{~mJ} / \mathrm{cm}^{2}$ or more as shown in Fig. 6.

Pullulan-A maintained a residual film ratio of $80 \%$ or more, regardless of photosensitizer wt $\%$ or $>75$ $\mathrm{mJ} / \mathrm{cm}^{2}$ exposure, confirming that it is a highly sensitive material (Fig. 7).

As shown in Fig. 5-7, the difference in molecular 
weight of each base polymer as shown in Fig. 1 has a great influence on its sensitivity.

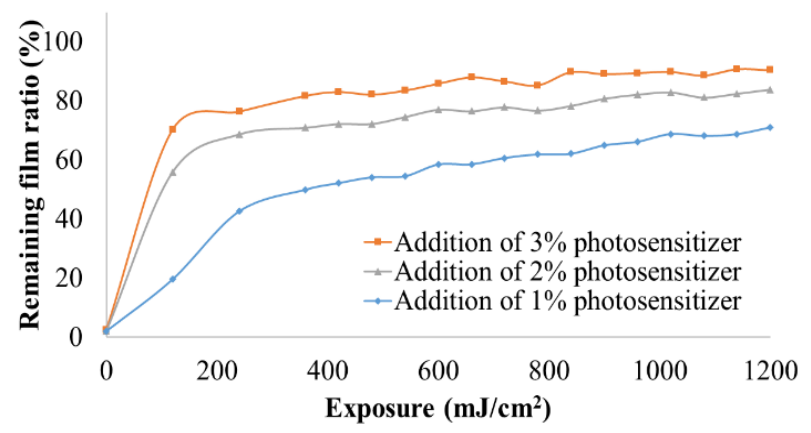

Fig. 5. NSD500-A sensitivity curve.

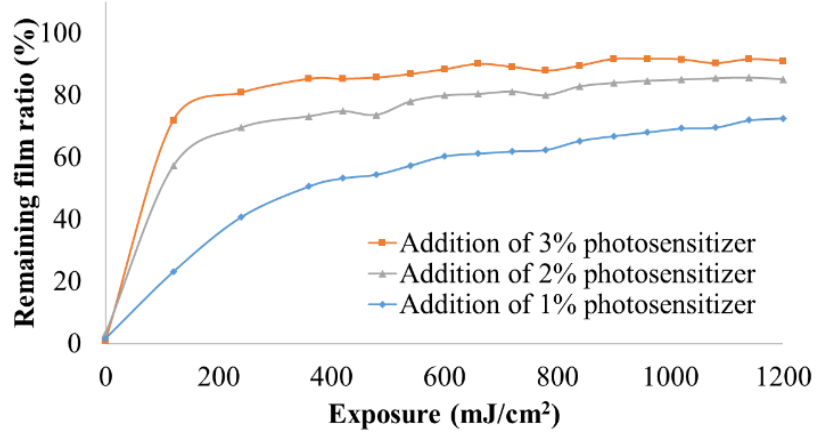

Fig. 6. NSD500Hi-A sensitivity curve.

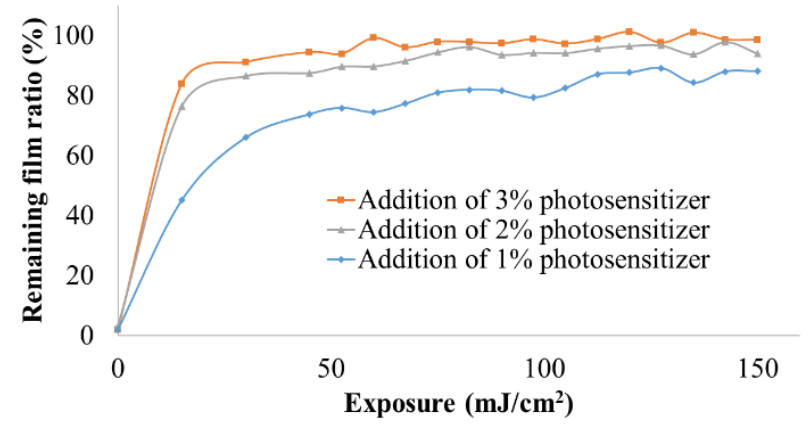

Fig .7. Pullulan-A sensitivity curve.

\subsection{Etching resistance evaluation}

Polysaccharides have low carbon density (according to the Ohnishi parameter) and relatively low etching resistance. It was measured whether the etching resistance was affected by the bonding mode and the molecular weight of the base polymer. Etching resistance was low for all polysaccharides, and the reference, Cresol Novolac, gave the best results (Fig. 8 and 9).

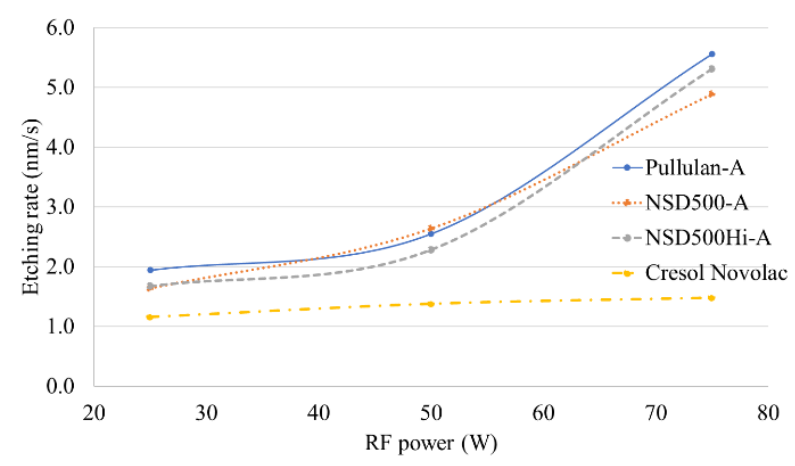

Fig. 8. Etching resistance evaluation result for $\mathrm{CF}_{4}$.

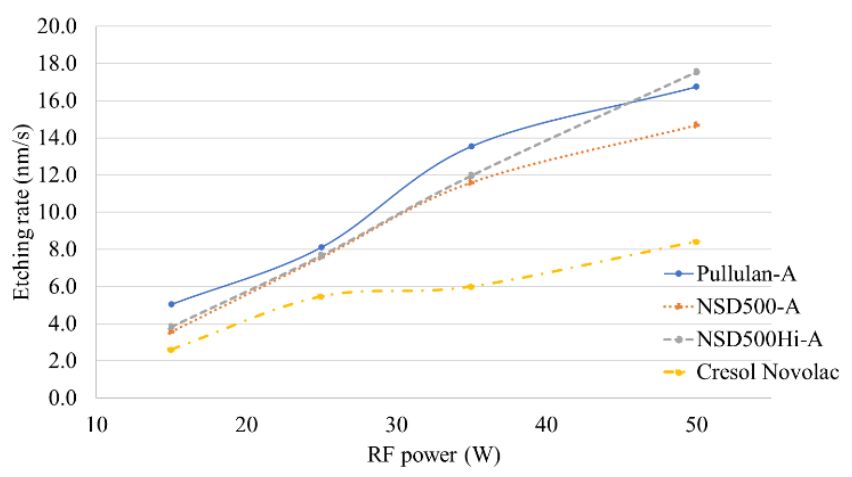

Fig. 9. Etching resistance evaluation result for $\mathrm{O}_{2}$.

Table 2. Ohnishi parameter calculation results.

\begin{tabular}{|c|c|c|c|c|}
\hline & Pullulan-A & NSD500-A & NSD500Hi-A & Cresol Novolac \\
\hline Structural formula & & $\mathrm{OH} /$ & & $\mathrm{CH}_{2}$ \\
\hline Ntotal & & $\mathrm{C}_{9} \mathrm{H}_{14} \mathrm{O}_{7}=30$ & & $\mathrm{C}_{8} \mathrm{H}_{10} \mathrm{O}_{1}=19$ \\
\hline Ncarbon & & 9 & & 8 \\
\hline Noxygen & & 7 & & 1 \\
\hline Ohnishi parameter & & 15.0 & & 2.7 \\
\hline
\end{tabular}


This result is consistent with commonly known Ohnishi parameter results.

The Ohnishi parameter is a formula that uses the difference in etching rate between carbon and oxygen and is expressed by the following formula:

$$
\underset{\text { parameter }}{\text { Ohnishi }}=\frac{\text { Ntotal }}{\text { Ncarbon }- \text { Noxygen }}
$$

where Ntotal is the total number of atoms in the molecule, Ncarbon is the number of carbon atoms in the molecule, and Noxygen is the number of oxygen atoms in the molecule.

For example, for benzene $\mathrm{C}_{6} \mathrm{H}_{6}$, Ntotal is 12 , Ncarbon is 6, Noxygen is 0 , and the Ohnishi parameter is 2.0. For phenol $\mathrm{C}_{6} \mathrm{H}_{6} \mathrm{O}_{1}$, Ntotal is 13, Ncarbon is 6 , and Noxygen is 1 ; thus, the Ohnishi parameter is 2.6. To increase the etching resistance, it is necessary to increase the carbon ratio and decrease the value of the Ohnishi parameter.

Table 2 lists calculations of the Ohnishi parameter for Pullulan-A, NSD500-A, NSD500Hi-A, and Cresol Novolac.

The Ohnishi parameter value for polysaccharides is large, whereas Cresol Novolac has a small value, which correlates with the results in Figs. 7 and 8.

\subsection{Coating film strength evaluation}

Pullulan exhibits coating strength that is not found in conventional water-soluble micropatterning polysaccharides because of its film-forming ability. Film strength measurements were also performed.

For the measurement samples, $3 \mathrm{wt} \%$ of photosensitizer was added to each polymer. Pullulan-A was exposed to $100 \mathrm{~mJ} / \mathrm{cm}^{2}$, and NSD500-A and NSD500Hi-A were exposed to $600 \mathrm{~mJ} / \mathrm{cm}^{2}$.

NSD500-A was determined to be soft, and Pullulan-A was hard based on the maximum displacement (hmax), indentation hardness, and indentation elastic modulus (Fig. 10).

This phenomenon correlates to exposure sensitivity and the residual film ratio. A material with good sensitivity and high residual film ratio is three-dimensionally crosslinked and has many polymerized portions.

Crosslinking and polymerization have a great influence on hardness (Table 3).

From the load and displacement curves shown in Fig. 10 and the values listed in Table 3, it was confirmed that there are no significant differences in hardness or elastic modulus between Pullulan-A and NSD500Hi-A.

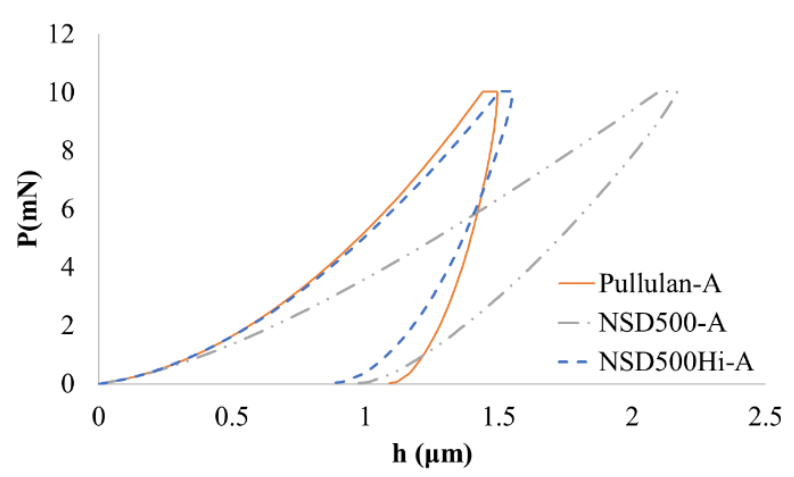

Fig. 10. Displacement measurement result for load by nano indenter.

Table 3. Hardness and elastic modulus measurements by nano indenter.

\begin{tabular}{|l|c|c|c|c|}
\hline & $\begin{array}{c}\text { Fmax } \\
(\mathrm{mN})\end{array}$ & $\begin{array}{c}\text { Hmax } \\
(\mu \mathrm{m})\end{array}$ & $\begin{array}{c}\text { Hit }^{\mathrm{a}} \\
(\mathrm{MPa})\end{array}$ & $\begin{array}{c}\text { Eit }^{\mathrm{b}} \\
(\mathrm{MPa})\end{array}$ \\
\hline Pullulan-A & 10.03 & 1.493 & 170.5 & $7.054 \mathrm{E}+03$ \\
\hline NSD500-A & 10.04 & 2.166 & 132.9 & $1.245 \mathrm{E}+03$ \\
\hline NSD500Hi-A & 10.04 & 1.553 & 188.2 & $3.679 \mathrm{E}+03$ \\
\hline
\end{tabular}

a) Hit is indentation hardness. b) Eit is indentation modulus.

Pullulan is an effective film former before UV curing, but after curing, it is not much different fromNSD500Hi-A, and pullulan's film-forming properties are lost due to three-dimensional crosslinking. It is suggested that UV curing adversely affects the film-forming ability.

\subsection{Microfabrication evaluation}

Microfabrication evaluation of the high molecular weight polysaccharide was carried out.

For the evaluation samples, $3 \mathrm{wt} \%$ of photosensitizer was added to each polymer. Pullulan-A was exposed to $100 \mathrm{~mJ} / \mathrm{cm}^{2}$, and NSD500-A and NSD500Hi-A were exposed to $600 \mathrm{~mJ} / \mathrm{cm}^{2}$.

NSD500-A and NSD500Hi-A showed good microfabrication results, and Pullulan-A had a lot of undissolved residue between the patterns.

NSD500-A is only slightly affected by UV reflection and diffraction during exposure because the base polymer is small molecular weight and the exposure sensitivity is low. Therefore, the uncured portion is easily dissolved in the developing solution (water), and relatively clean and fine patterning is possible. (Fig. 11).

NSD500Hi-A has the low molecular weight fraction removed, but its molecular weight is still relatively small, and its exposure sensitivity is not so high. Therefore, relatively clean and fine patterning was also possible (Fig. 12). 
Pullulan-A has a molecular weight 10 times or more than that of NSD500-A and an exposure sensitivity of 10 times or more.

Therefore, if there is even a small amount of UV reflection or diffraction between patterns that are not exposed to UV, the crosslinking reaction proceeds, and the polymer becomes insoluble in the developing solution (water), making microfabrication difficult. (Fig. 13).



Fig. 11. NSD500-A micropatterning results.



Fig. 12. NSD500Hi-A micro patterning results.

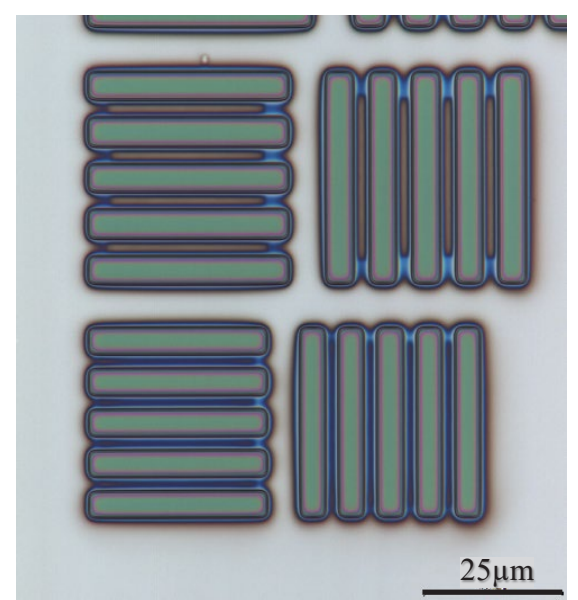

Fig. 13. Pullulan-A micropatterning results.

\section{Conclusion}

The effectiveness of water-soluble micropatterning materials using high molecular weight polysaccharides was investigated. By converting the hydroxyl groups of the high molecular weight polysaccharide into photosensitive groups to the extent that water solubility can be maintained, a water-soluble micropatterning material was created. The obtained water-soluble micropatterning material had better film-forming properties before UV curing and higher sensitivity than those using dextrins. However, effectiveness of the coating films after UV curing could not be confirmed because it was not much different from that of dextrins. Exposure sensitivity was too high, and even a small amount of UV reflection or diffraction caused the undissolved residue between the patterns, making fine patterning difficult.

It will be necessary to optimize this material by adjusting the amount and concentration of the photosensitizer added to use it as a water-soluble micropatterning material.

\section{References}

1. H. Morishita, M. Ito, N. Hayashi, and S. Nonogaki, J. Photopolym. Sci. Technol., 7 (1994) 59.

2. Q. Lin, T. Steinhausler, L. Simpson, M. Wilder, D. R. Medeiros, and C. G. Willson, Chem. Mater., 9 (1997) 1725.

3. J. M. Havard, M. Yoshida, D. Pasini, N. Vladimirov, J. M. J. Frechet, D.R. Medeiros, K. Patterson, S. Yamada, C. G. Willson, J. D. Byers, J. Polym. Sci.: Part A: Polymer Chemistry, 37 (1999) 1225.

4. J. M. Havard, S.-Y. Shim, and J. M. J. Frechet, Chem. Mater., 11 (1999) 719.

5. K. Kojima, M. Ito, H. Morishita, and N. Hayashi, Chem. Mater., 10 (1998) 3429.

6. J. Swei and J. B. Talbot, J. Appl. Polym. Sci., 102 (2006) 1637.

7. S.-Y. Shim and J.-M. Kim, Bull. Korean Chem. Soc., 22 (2001) 1120.

8. K. H. Chae, G. J. Sun, J. K. Kang, and T. H. Kim, J. Appl. Polym. Sci., 86 (2002) 1172.

9. Y. Y. Liao and J.-H. Liu, J. Appl. Polym. Sci., 109 (2008) 3849.

10. T. Amano, D. Hirata, Y. Hasegawa, and S. Takei, J. Photopolym. Sci. Technol., 33 (2020) 445. 